\title{
SER-CAT Scientific Highlights and Beamline Upgrade \& User Program During the APS Shutdown
}

\author{
Bi-Cheng Wanga, John Rose ${ }^{\mathrm{b}}$ and John Chrzas ${ }^{\mathrm{c}}$
}

SER-CAT, Department of Biochemistry and Molecular Biology, University of Georgia, Athens, GA and the Advanced Photon Source, Argonne National Laboratory, Argonne, IL

abcwang@uga.edu

bjprose@uga.edu

chrzas@anl.gov

SER-CAT (SouthEast Regional Collaborative Access Team) is a consortium of 22-member institutions and five Associate User groups. It was established in 1997 for the construction and operation of two macromolecular beamlines at the Advanced Photon Source (APS), Argonne National Laboratory. SER-CAT's productivity is outstanding. It continually ranks among the top beamlines at the APS (and worldwide) in terms of annual peer reviewed publications and PDB deposits. It is the 2 nd most productive beamline (in terms of PDB entries) at the APS as well as in the world!

The APS Upgrade (APS-U) is a large scale project to replace the existing APS storage ring lattice with a Multiple Bend Achromat (MBA) lattice at an estimated cost of 0.77 billion dollars (https://www.aps.anl.gov/APS-Upgrade). To accomplish this project, the APS will be shut down for a minimum of 1-2 year starting in early 2022. The SER-CAT Board views the 2022 APS shutdown period as an opportunity and has set the priorities to upgrade the optics, electronics and end-station hardware of the current ID beamline (22ID-D) to best use the very small, symmetrically focused intense beams made possible by the new MBA lattice. In addition, plans are underway to construct a second ID beamline (22ID-E) at Sector 22.

In this presentation, both the SER-CAT current scientific highlight and planned beamline upgrade and user services during the APS shutdown period will be introduced and discussed. 